\title{
A GEOMETRIC PROPERTY OF THE ROOTS OF CHEBYSHEV POLYNOMIALS
}

\author{
Roman Wituła, Edyta Hetmaniok, Damian Słota \\ Institute of Mathematics, Silesian University of Technology \\ Gliwice, Poland \\ Edyta.Hetmaniok@polsl.pl
}

\begin{abstract}
In this text a new property of geometric nature of the Chebyshev polynomials is given. It is proven that the lengths of diagonals of a regular $n$-gon with the side of length equal to one are the sums of positive roots of the respective renormalized Chebyshev polynomials of one from among four types. Some new special decompositions of differences of values of the Chebyshev polynomials are also presented.
\end{abstract}

Keywords: Chebyshev polynomials, Vieta-Lucas polynomials

AMS Subject Classification: 26C05, 11B83, 12D10.

\section{Introduction}

The aim of this short text is to present one more fundamental property of Chebyshev polynomials related to the geometric-analytical nature of their roots. We want to emphasize that this property seems to be completely original.

Inspired by paper [1], we have noticed a slightly deeper relationship between the length of diagonals of the regular $n$-gons and the sums of positive roots of any of all four types of Chebyshev polynomials (see [2-4]):

$$
\begin{aligned}
& \text { 1st kind } \quad \boldsymbol{T}_{n}(\cos x)=\cos (n x), \quad x \in \mathbb{R} \text {, } \\
& \text { 2nd kind } \quad \boldsymbol{U}_{n}(\cos x)=\frac{\sin ((n+1) x)}{\sin x}, \quad x \in \mathbb{R} \backslash \pi \mathbb{Z} \text {, } \\
& 3 r d \text { kind } \quad \boldsymbol{V}_{n}(\cos x)=\frac{\cos \left(\left(n+\frac{1}{2}\right) x\right)}{\cos \frac{x}{2}}, \quad x \in \mathbb{R} \backslash \pi(2 \mathbb{Z}+1) \text {, } \\
& 4 \text { th kind } \quad \boldsymbol{W}_{n}(\cos x)=\frac{\sin \left(\left(n+\frac{1}{2}\right) x\right)}{\sin \frac{x}{2}}, x \in \mathbb{R} \backslash 2 \pi \mathbb{Z} \text {, }
\end{aligned}
$$

for every $n \in \mathbb{N} \cup\{0\}$. For the reasons of cosmetic nature it is better to consider the re-scaling Chebyshev polynomials 


$$
\begin{aligned}
& \boldsymbol{T}_{n}^{*}(x):=2 \boldsymbol{T}_{n}\left(\frac{x}{2}\right), \quad \boldsymbol{U}_{n}^{*}(x):=\boldsymbol{U}_{n}\left(\frac{x}{2}\right), \quad \boldsymbol{V}_{n}^{*}(x):=\boldsymbol{V}_{n}\left(\frac{x}{2}\right), \\
& \text { and } \boldsymbol{W}_{n}^{*}(x):=\boldsymbol{W}_{n}\left(\frac{x}{2}\right), \quad n \in \mathbb{N} .
\end{aligned}
$$

We note that polynomials $\boldsymbol{T}_{n}^{*}(x)$ are called either Vieta-Lucas polynomials $[5,6]$ or Dickson's polynomials [7]. Whereas $\boldsymbol{U}_{n}^{*}(x)$ are called Vieta-Fibonacci polynomials [6]. Properties of algebraic and combinatoric nature of these polynomials are discussed for example in papers [5-11]. One of the spectacular properties of $\boldsymbol{T}_{n}^{*}(x)$ are the following decompositions proved in [6]:

$$
\begin{gathered}
\boldsymbol{T}_{2 n-1}^{*}(x)-\boldsymbol{T}_{2 n-1}^{*}\left(\theta+\theta^{-1}\right)=\boldsymbol{T}_{2 n-1}^{*}(x)-\theta^{2 n-1}-\theta^{-2 n+1}= \\
=\prod_{k=0}^{2 n-2}\left(x-\theta \xi^{2 k}-\theta^{-1} \xi^{-2 k}\right),
\end{gathered}
$$

where $\xi=\exp \left(\frac{i \pi}{2 n-1}\right)$;

$$
\begin{aligned}
(-1)^{n} \boldsymbol{T}_{2 n}^{*}(i x) & +\boldsymbol{T}_{2 n}^{*}\left(\theta+\theta^{-1}\right)=(-1)^{n} \boldsymbol{T}_{2 n}^{*}(i x)+\theta^{2 n}+\theta^{-2 n}= \\
& =\prod_{k=0}^{2 n-1}\left(x-\theta \zeta^{2 k+1}+\theta^{-1} \zeta^{-2 k-1}\right)
\end{aligned}
$$

where $\zeta=\exp \left(\frac{i \pi}{2 n}\right)$, for every $n \in \mathbb{N}, \theta \in \mathbb{C}$ and $\theta \neq 0$. For example, we obtain the following special ones

$$
\begin{aligned}
& \boldsymbol{T}_{2 n-1}^{*}(x)-2 \cos ((2 n-1) \varphi)=\prod_{k=0}^{2 n-2}\left(x-2 \cos \left(\varphi+\frac{2 k \pi}{2 n-1}\right)\right), \\
& \boldsymbol{T}_{2 n-1}^{*}(x)-\boldsymbol{T}_{2 n-1}^{*}(2 \csc \varphi)=\boldsymbol{T}_{2 n-1}^{*}(x)-\tan ^{2 n-1}\left(\frac{\varphi}{2}\right)-\cot ^{2 n-1}\left(\frac{\varphi}{2}\right)= \\
&=\prod_{k=0}^{2 n-2}\left(x-2 \csc \varphi \cos \frac{2 k \pi}{2 n-1}+2 i \cot \varphi \sin \frac{2 k \pi}{2 n-1}\right),
\end{aligned}
$$

for $\varphi \neq k \pi, k \in \mathbb{Z}$

$$
\begin{aligned}
& (-1)^{n} \boldsymbol{T}_{2 n}^{*}(i x)+\boldsymbol{T}_{2 n}^{*}(2 \sec \alpha)= \\
& =(-1)^{n} \boldsymbol{T}_{2 n}^{*}(i x)+\tan ^{2 n}\left(\frac{\pi}{4}-\frac{\alpha}{2}\right)+\tan ^{2 n}\left(\frac{\pi}{4}+\frac{\alpha}{2}\right)= \\
& =\prod_{k=0}^{2 n-1}\left(x+2 \tan \alpha \cos \frac{(2 k+1) \pi}{2 n}-2 i \sec \alpha \sin \frac{(2 k+1) \pi}{2 n}\right)
\end{aligned}
$$


for $\alpha \neq \frac{\pi}{2}+k \pi, k \in \mathbb{Z}$,

$$
\begin{aligned}
& (-1)^{n} \boldsymbol{T}_{2 n}^{*}(i x)+\boldsymbol{T}_{2 n}^{*}(\operatorname{coth} \alpha)= \\
& =(-1)^{n} \boldsymbol{T}_{2 n}^{*}(i x)+\operatorname{coth}^{2 n}\left(\frac{\alpha}{2}\right)+\tanh ^{2 n}\left(\frac{\alpha}{2}\right)= \\
& =\prod_{k=0}^{2 n-1}\left(x+2 \operatorname{csch} \alpha \cos \frac{(2 k+1) \pi}{2 n}-2 i \operatorname{coth} \alpha \sin \frac{(2 k+1) \pi}{2 n}\right),
\end{aligned}
$$

for $\alpha \neq 0$.

Other fundamental properties, especially of an analytic nature, of polynomials $\boldsymbol{T}_{n}^{*}, \boldsymbol{U}_{n}^{*}, \boldsymbol{V}_{n}^{*}$ and $\boldsymbol{W}_{n}^{*}$ are presented in monographs [2,4], see also interesting new results on a moment problem [12].

\section{Main result}

Positive roots of polynomials $\boldsymbol{T}_{n}^{*}, \boldsymbol{U}_{n}^{*}, \boldsymbol{V}_{n}^{*}$ and $\boldsymbol{W}_{n}^{*}$, respectively, are listed below [2]:

$$
\begin{array}{ll}
t_{k, n}=2 \cos \left(\frac{(2 k-1) \pi}{2 n}\right), & k=1,2, \ldots,\left\lfloor\frac{n}{2}\right\rfloor, \\
u_{k, n}=2 \cos \left(\frac{k \pi}{n+1}\right), & k=1,2, \ldots,\left\lfloor\frac{n}{2}\right\rfloor, \\
v_{k, n}=2 \cos \left(\frac{(2 k-1) \pi}{2 n+1}\right), & k=1,2, \ldots,\left\lfloor\frac{n+1}{2}\right\rfloor, \\
w_{k, n}=2 \cos \left(\frac{2 k \pi}{2 n+1}\right), & k=1,2, \ldots,\left\lfloor\frac{n}{2}\right\rfloor,
\end{array}
$$

(we note that zeros $t_{k, n}, k=1,2, \ldots,\left\lfloor\frac{n}{2}\right\rfloor$, could also be deduced from (1) for $\theta=\exp \left(\frac{i \pi}{4 n-2}\right)$ and from (2) for $\theta=\exp \left(\frac{i \pi}{4 n}\right)$ ).

We will present now our main result.

Theorem. Let $n \in \mathbb{N}, n \geq 4$, and $V_{0}, V_{1}, \ldots, V_{n-1}$ denote the vertices of a regular $n$-gon $P$ with the side of length 1 . If $x=\frac{\pi}{n}$ and $d\left(V_{k}, V_{l}\right)$ denotes the distance between $V_{k}$ and $V_{l}$, then we have

(i)

$$
d\left(V_{0}, V_{m}\right)=\frac{\sin (m x)}{\sin x}=\boldsymbol{U}_{m-1}(\cos x)
$$

for $1 \leq m \leq\left\lfloor\frac{n}{2}\right\rfloor$, 
(ii)

$$
d\left(V_{0}, V_{m+1}\right)-d\left(V_{0}, V_{m}\right)=\frac{\cos \left(\left(m+\frac{1}{2}\right) x\right)}{\cos \frac{x}{2}}=V_{m}(\cos x),
$$

for $1 \leq m+1 \leq\left\lfloor\frac{n}{2}\right\rfloor$,

(iii)

$$
d\left(V_{0}, V_{m+1}\right)-d\left(V_{0}, V_{m-1}\right)=2 \cos (m x)=2 \boldsymbol{T}_{m}(\cos x),
$$

for $2 \leq m+1 \leq\left\lfloor\frac{n}{2}\right\rfloor$,

(iv)

$$
d\left(V_{0}, V_{2 m}\right)=2 \sum_{k=1}^{m} \cos ((2 k-1) x)
$$

for $2 \leq 2 m \leq\left\lfloor\frac{n}{2}\right\rfloor$,

(v)

$$
d\left(V_{0}, V_{2 m+1}\right)=1+2 \sum_{k=1}^{m} \cos (2 k x),
$$

for $1 \leq 2 m+1 \leq\left\lfloor\frac{n}{2}\right\rfloor$.

Corollary. By comparing the terms of sums from identities $(i v)$ and $(v)$ with the roots of all four types of rescaling Chebyshev polynomials $\boldsymbol{T}_{n}^{*}, \boldsymbol{U}_{n}^{*}, \boldsymbol{V}_{n}^{*}$ and $\boldsymbol{W}_{n}^{*}$, we obtain

for $2 \leq 2 m \leq\left\lfloor\frac{n}{2}\right\rfloor$ and

$$
d\left(V_{0}, V_{2 m}\right)= \begin{cases}\sum_{k=1}^{m} t_{k, r} & \text { if } n=2 r, \\ \sum_{k=1}^{m} v_{k, r} & \text { if } n=2 r+1,\end{cases}
$$

$$
d\left(V_{0}, V_{2 m+1}\right)= \begin{cases}1+\sum_{k=1}^{m} w_{k, r} & \text { if } n=2 r+1, \\ 1+\sum_{k=1}^{m} u_{k, r} & \text { if } n=2(r+1),\end{cases}
$$

for $1 \leq 2 m+1 \leq\left\lfloor\frac{n}{2}\right\rfloor$. 
Proof of Theorem. If $r$ denotes the radius of the circle circumscribed on $P$, then by the law of sines we get $r=\frac{1}{2 \sin x}$ and $d\left(V_{0}, V_{m}\right)=\frac{\sin (m x)}{\sin x}, m \leq\left\lfloor\frac{n}{2}\right\rfloor$. Hence we obtain

$$
\begin{aligned}
& d\left(V_{0}, V_{m+1}\right)-d\left(V_{0}, V_{m}\right)=\frac{\sin ((m+1) x)-\sin (m x)}{\sin x}= \\
& =\frac{2 \sin \frac{x}{2} \cos \left(\left(m+\frac{1}{2}\right) x\right)}{\sin x}=\frac{\cos \left(\left(m+\frac{1}{2}\right) x\right)}{\cos \frac{x}{2}}=V_{m}(\cos x),
\end{aligned}
$$

for $1 \leq m+1 \leq\left\lfloor\frac{n}{2}\right\rfloor$, and next we have

$$
\begin{aligned}
& d\left(V_{0}, V_{m+1}\right)-d\left(V_{0}, V_{m-1}\right)= \\
& =\left(d\left(V_{0}, V_{m+1}\right)-d\left(V_{0}, V_{m}\right)\right)+\left(d\left(V_{0}, V_{m}\right)-d\left(V_{0}, V_{m-1}\right)\right)= \\
& =\frac{\cos \left(\left(m+\frac{1}{2}\right) x\right)+\cos \left(\left(m-\frac{1}{2}\right) x\right)}{\cos \frac{x}{2}}= \\
& =\frac{2 \cos (m x) \cos \frac{x}{2}}{\cos \frac{x}{2}}=2 \cos (m x)=2 \boldsymbol{T}_{m}(\cos x),
\end{aligned}
$$

for $2 \leq m+1 \leq\left\lfloor\frac{n}{2}\right\rfloor$.

The last identity implies

$$
d\left(V_{0}, V_{2 m}\right)=\sum_{k=1}^{m}\left(d\left(V_{0}, V_{2 k}\right)-d\left(V_{0}, V_{2(k-1)}\right)\right)=2 \sum_{k=1}^{m} \cos ((2 k-1) x),
$$

for $2 \leq 2 m \leq\left\lfloor\frac{n}{2}\right\rfloor$, and

$$
d\left(V_{0}, V_{2 m+1}\right)=1+\sum_{k=1}^{m}\left(d\left(V_{0}, V_{2 k+1}\right)-d\left(V_{0}, V_{2 k-1}\right)\right)=1+2 \sum_{k=1}^{m} \cos (2 k x),
$$

for $2 \leq 2 m+1 \leq\left\lfloor\frac{n}{2}\right\rfloor$, which ends the proof.

\section{Conclusions}

In the paper we have described the new property of zeros of renormalized Chebyshev polynomials. It has been proven that the lengths of diagonals of a regular $n$-gon with the side of length equal to one are the sums of positive roots of the 
respective renormalized Chebyshev polynomials of one from among four types. Formulae for decompositions of differences of values of the Chebyshev polynomials have been presented as well.

\section{References}

[1] Bialostocki A., Bialostocki D., Fluster M., Holman W., McAleer M., A geometric property of renormalized Chebyshev polynomials, College Math. J. (in review).

[2] Mason J.C., Handscomb D.C., Chebyshev Polynomials, Chapman \& Hall/CRC, New York 2003.

[3] Paszkowski S., Numerical Applications of Chebyshev Polynomials and Series, PWN, Warsaw 1975 (in Polish).

[4] Rivlin T., Chebyshev Polynomials from Approximation Theory to Algebra and Number Theory, 2nd ed., Wiley, New York 1990.

[5] Robbins N., Vieta's triangular array and a related family of polynomials, Internat. J. Math. Math. Sci. 1991, 14, 239-244.

[6] Wituła R., Słota D., On modified Chebyshev polynomials, J. Math. Anal. Appl. 2006, 324, 321-343.

[7] Bayad A., Cangul I.N., The minimal polynomial of $2 \cos (\pi / q)$ and Dickson polynomials, Appl. Math. Comput. 2012, 218, 7014-7022.

[8] Knopfmacher A., Mansour T., Munagi A., Prodinger H., Staircase words and Chebyshev polynomials, Appl. Anal. Discrete Math. 2010, 4, 81-95.

[9] Wituła R., Hetmaniok E., Słota D., Prodinger's algebraic identities and their applications, Int. J. Pure Appl. Math. 2010, 64, 225-237.

[10] Wituła R., Słota D., Decompositions of certain symmetric functions of powers of cosine and sine functions, Int. J. Pure Appl. Math. 2009, 50, 1-12.

[11] Zhang Z., Wang J., On some identities involving the Chebyshev polynomials, Fibonacci Quart. 2004, 42, 245-249.

[12] Castillo K., Lamblem R.L., Sri Ranga A., On a moment problem associated with Chebyshev polynomials, Appl. Math. Comput. 2012, 218, 9571-9574. 